\title{
Good Things Come to Those Who Binge: An Exploration of Binge-Watching Related Behavior
}

\author{
Paul BOCA, PhDc \\ Department of Journalism and Digital Media \\ Faculty of Political, Administrative and Communication Sciences \\ Babeș-Bolyai University, Cluj-Napoca, Romania \\ E-mail: boca@fspac.ro
}

\begin{abstract}
The rise of Video-on-Demand (VoD) services on the media market has produced key mutations in the television series industry at all levels. The dynamics of the tripartite relation between production, distribution and consumption gained new forms when these new services broke the traditional lineage of television and adopted the aesthetics of media catalogs that users can access whenever and wherever they want. VoD portals have triggered a process of usage democratization that has led to the unprecedented popularization of binge-watching as a new norm for the consumption of TV series. The present study explores binge-watching as a way of spending leisure time and the motivations behind the users' decisions to engage in extended viewing sessions. Certain specific behaviors and emotions related to marathon viewing are discussed, as well as the connection between genre and extended watching sessions or users' general attitude towards binge-watching.
\end{abstract}

Keywords: Binge-watching; Netflix; Netflix original series; Videoon-Demand; Social networking sites. 


\section{Introduction}

The advent of online streaming services such as Netflix, Hulu or Amazon Prime Video has changed the distribution and consumption patterns of TV series (Hiller, 2017; Jenner, 2015, 1; Lotz, 2014, 5). By implementing new ways in which media products reach consumers, the Video-on-Demand $(\mathrm{VoD})$ portals have refreshed the way audiences perceive the very concept of television.

VoD portals offer users access to a vast catalog of productions based on a prepaid monthly subscription. The main difference between traditional, linear TV consumers and $\mathrm{VoD}$ subscribers stands in the fact that the latter are able to watch what, when, where and how they want. Thus, from a consumer who used to spend his whole day on the couch, watching a sequence of programs broadcast by a certain television station, or zapping through tens or hundreds of channels, the industry has reached a new stage in its evolution, whose epitome is a permanently connected user, who is able to access a huge array of media products at any time, from anywhere. This business model - which relies on catalogs or bundles, rather than broadcasting a linear sequence of productions - has also emerged in music distribution services such as Spotify or Deezer, as well as in the book industry, and revolves around a transfer of power from the distributor to the user.

In this context, the distribution mechanisms used by Subscription Video-onDemand (SVoD) services lead to the emergence of a media marathon culture (Wayne, 2014). The most visible manifestation of this phenomenon is bingewatching, a consumer behavior defined by Netflix Media Center (2013) as viewing two to six episodes of the same production in a single sitting. Since 2013, when the term 'binge-watching' made it to the Oxford Dictionaries Word of the Year short list (Oxford Dictionaries, 2013), this type of behavior has had a central role in the lives of many consumers and, beyond that, in the global agenda of the television production industry.

On the one hand, the phenomenon is boosted by the distribution system itself - Netflix, for example, publishes all the episodes of a season at once, which gives subscribers the opportunity to watch the productions at their own pace. On the other hand, the explanation lies in the fact that the multi-episodic distribution gives creators more freedom: keeping in mind that, statistically speaking (Shannon-Missal, 2013), most users will watch multiple episodes of a production in a single sitting, screenwriters, as well as directors and producers can focus on the overall development of the fictional world, rather than struggling to create episodes which can - at least up to a certain point - work individually. From this point of view, binge-watching inevitably implies that consumers will have a much fresher memory of the narrative world than weekly production fans. Consequent$l y$, the changing prerequisites that digital series' creators face lead to their ability to put into practice more complex narratives (Lotz, 2017; Mittell, 2015), focusing 
on the story's progression in time - or longer narrative arcs - and multidimensional characters.

These changes in the discursive patterns of TV series, as well as the technological developments that made new narrative formulas viable for the market, have all led to a media landscape where binge-watching is adopted as a mainstream media practice (Jenner, 2015, 1-3). According to recent surveys, 75\% of American consumers and $85 \%$ of Chinese consumers using $\mathrm{VoD}$ services engage in bingewatching television shows (ARRIS Consumer Entertainment Index, 2015).

In this context, this paper aims to explore behavioral and emotional patterns related to binge-watching digital series through Netflix, the biggest VoD player on the market. Although the motivations for binge-watching have already been examined through surveys by several papers (Conlin, Billings, \& Auverset, 2016; Merikivi, Mäntymäki, Salovaara, \& Zhang, 2016; Pittman \& Sheehan, 2015; Steiner \& Xu, 2018; Walton-Pattison, Dombrowski, \& Presseau, 2016), the novelty of the present article stands in the fact that it does so by automatically analyzing a large data set comprised of comments related to binge-watching posted by users on the official Facebook pages of 71 original Netflix productions. Choosing user generated comments as a starting point has revealed some additional interesting behaviors and emotions that people tend to associate with their binge-watching sessions when it comes to their self-representation on social networking sites. As Netflix's revenue has been continuously rising during the last decade (MacroTrends, 2019), it has become one of the most powereful internet companies worldwide (Statista, 2018) and it has invested almost 6.8 billion dollars in original content in 2018 alone (Deloitte, 2019). Keeping in mind the popular connection between binge-watching as a media craze and consumption phenomenon and Netflix, it is important to study this type of behavior in its direct relation to its source. This paper examines which kind of shows usually gets associated with binge-watching, what are the main user behavior patterns related to this type of media consumption and the network of connections between different behaviors and the emotions generated by them. Moreover, the paper aims to evaluate the general attitude of the audience toward binge-watching.

\section{Theoretical framework}

VoD portals liberate consumers from the constraints of linear television. The new television is about freedom of choice and mobility, and is based on a user-centered consumption mechanism that is closely related to the distribution mode. Ted Sarandos, Netflix's chief content officer, says the company's decision to release all the episodes of a season simultaneously came from a practical rationale: it was the way Netflix, who had not produced original content until 2013, when it released the first season of House of Cards, was accustomed to publishing the productions for which they had purchased the broadcasting rights. On the other hand, he says, 
the US company already had data on how Netflix users were consuming the content: Some watched four episodes, others watched two. Some of them watched seven. No one watched one. (The Aspen Institute, 2015).

With the publication of whole seasons instead of weekly episodes, a strategy that later became known as "all-at-once" (Welch, 2013; West, 2014) or the "Netflix model" (Karve, 2018; Sweney, 2018), the American company managed to create a form of distribution that would play an important role in the early years of posttelevision.

\section{Binge-watching}

Chuck Tryon $(2015,105)$ says one of the key moments that defined bingewatching as a new norm for the consumption of TV series was the publication by Wired Magazine of an extended advertorial for Netflix under the title TV Got Better. In this multimedia article, anthropologist Grant McCracken "recoded the practice of 'binge viewing' as 'feasting', a phrasing that recasts the practice of watching TV as healthy, as a good form of consumption" (Tryon, 2015, 105). Thus, new technologies for television distribution and consumption are seen as sources of democratization and legitimacy, as opposed to traditional television, as a medium of very limited choice. To put it in other words, "In contrast to its previous status, in the era of convergence, television has significantly improved its position in the cultural hierarchy" (Newman, 2014, 68). In this sense, the concept of binge-watching has lost the negative connotations of similar expressions such as "binge-drinking" or "binge-eating", which denounce self-destructive behaviors, associated with diseases such as alcoholism or bulimia (Stoldt, 2013, 18).

Chuck Tryon $(2015,105)$ emphasizes the fact that the discourse through which Netflix attempts to define itself as being different from linear TV is built on the binary opposition between the passive traditional television and the active web. According to him, the gradual positioning of online media consumption as an active one, at the opposite of watching linearly distributed television programs, marks an important moment in redefining the TV medium.

Steiner and $\mathrm{Xu}$ (2018) argue that the distinction between passive and active consumption, predominant in the literature of recent decades, is imprecise and propose a different classification of consumption types, centered on the degree of attention that users pay when watching media products. Beyond this observation, it is clear that the new type of consumption made possible by Subscription Video-on-Demand $(\mathrm{SVoD})$ portals revolves around the user, who has the power to choose when, where, and especially how much he watches. Therefore, bingewatching is not the new consumer norm (Netflix Media Center, 2013) exclusively due to an industry decision, as in the case of traditional television, but rather due to the choices made by users. 
The concept of binge-watching is not easy to define. A survey showed that $73 \%$ of Netflix users associate this type of consumption with watching two to six episodes of the same production in a single sitting (Netflix Media Center, 2013). On the other hand, referring to the consumption of series on DVD box-sets, Debra Ramsey (cited in Jenner, 2015) says that binge-watching is associated with the consumption of a whole season in one day, while Nolan Feeney (2014) states that binge-viewing starts after you have watched 2 or 3 hours of the same show continuously.

Critic Mary McNamara (2012) gave her own definition of the term: "Any instance where more than three one-hour episodes or six half-hour comedy episodes are consumed in one session". On the other hand, the term binge-watch has also been defined as "watching multiple episodes (of a television program) in a fast succession, typically on DVDs or by digital streaming" (Oxford English Dictionaries, n.d.), without reference to a precise timespan.

If we refer to the meaning of the term "binge," that of "consumption in excess", it is difficult to accurately identify what would excess mean in this case, as long as there is currently no norm in this regard. In traditional television, the norm is one episode per week. If we follow this logic, "excess" may even mean one or two episodes per day (Jenner, 2015). From this perspective, it is difficult to apply the same norm to mediums whose mechanisms differ so much. Even though there is no unanimously accepted definition (data provided by Netflix Media Center tends to be the most cited by scholars), it is certain that binge-watching has had - since 2013 - a fundamental role in redefining the term television as we understand it today. The chance that such a phenomenon arose is related to the transfer of control power from the industry to the user. We can not talk about binge-watching without the so-called "autonomous programming" of consumption (Jenner, 2015).

According to Chuck Tryon $(2015,106)$, SVoD portals encourage the sequential consumption of several segments of the same series in a single sitting by distributing media products in bundles, through catalogs of media products that can be consumed in ways that are fundamentally different from the mechanisms of linear television.

In the same vein, Mareike Jenner (2015) talks about binge-watching as the central strategy of SVoD services. According to her, economically speaking, Netflix aims at avoiding subscription interruptions. As long as consumers engage in media marathons, the chances for them to drop off their subscription decrease exponentially. From this perspective, Netflix largely builds its distribution strategy around binge-watching, relying on an all-at-once distribution system and personalized user recommendations (Jenner, 2015). The great focus on this type of consumption in Netflix's strategy is also visible in the use of the phrase "bingeworthy" in the customized recommendation menu given by the streaming service. 
Mareike Jenner (2015) examines the causal connection between Netflix's official data, which shows that the average user of the portal considers a session of between two to six episodes of the same production binge-watching and the company's economic strategy. The author says that as long as the sequential consumption of only two episodes allows people to be part of the major cultural trend of binge-watching, it will be harder for them to give up their Netflix subscription. The mechanism also explains the adoption of similar distribution strategies by other prominent companies such as Hulu or Amazon (Jenner, 2015).

Chuck Tryon $(2015,106)$ notes that choosing to consume media products as soon as they are available in $\mathrm{VoD}$ catalogs is related to the desire of people to gain "cultural capital," that is to be among the first ones to participate in the discussions about a particular series through social networking sites (SNS) such as Twitter or Facebook. The phenomenon is not entirely new. In the case of linearly distributed television, fans are encouraged to watch shows when they are running for the first time, in tune with a similar social mechanism. The simultaneous distribution of all episodes in a season, on the other hand, gives users the opportunity to watch episodes one after another or to spread them for a longer period of time, according to their own schedule.

However, services like Netflix mimic the broadcasting experience of linear television by promoting the idea that users will be left behind if they do not watch a production as soon as it is available in the catalog (Tryon, 2015, 107). The complex motivations that make consumers engage in prolonged watching sessions will be examined in the next subchapter.

\section{Uses and gratifications in binge-watching}

Several studies (Conlin et al., 2016; Merikivi et al., 2016; Pittman \& Sheehan, 2015; Steiner \& Xu, 2018; Walton-Pattison et al., 2016) address the issue of bingewatching from the perspective of the uses and gratifications theory. Such papers largely follow the theoretical framework developed by Katz, Blumler and Gurevitch (1974). In the past, similar research has been applied to the consumption of linear television, with a focus on psychological motivations and the social component of consumers' behavior. Alan M. Rubin (1983) identifies nine main motivations that lead to the consumption of TV programs: relaxation, the feeling of company that an open TV provides, entertainment, social interaction, information, habit, time occupation, sexual arousal and escapism. Speaking about consumers' behavior in relation to reality shows, Papacharissi and Mendelson (2007) say, following the results of other studies published in the early 2000s, that voyeurism also plays an important role in people's decision to watch these productions. At the same time, they show that watching TV shows has penetrated people's lives so much that it has gained the accents of a ritual. 
Researches carried in the light of the uses and gratifications theory start from the premise of an active consumption, where the audience public has the initiative to select and use the technologies at its disposal to meet certain needs or desires, under the influence of social and psychological factors (Papacharissi \& Mendelson, 2007, 356). From the perspective of active consumption, Levy and Windahl (1984) have emphasized the importance of an approach that takes into account audiences' behavior pre-, post- and during exposure to productions ("Preactivity", "Postactivity", "Duractivity"). An approach on binge-watching from the perspective of the uses and gratifications theory is all the more important because this type of consumption is closely related to an active behavior, where the power of control stands in users' hands.

Steiner and $\mathrm{Xu}$ (2018) show that the main reasons why users choose to engage in binge-watching sessions are catching up with the missed episodes, relaxation, the feeling of fulfillment that users have after completing a series or season, immersion in the narrative universe, cultural inclusion and a better consumer experience. Also, qualities such as portability and navigability (the ability to control navigation through episodes using the pause, playback or fast forward functions) play an important role in consumers' decision to watch multiple episodes in a single sitting.

The study also reveals the contradictions in this type of consumption: on the one hand, users feel shame, guilt and regret about their prolonged binge-watching sessions, and on the other, they feel proud and connected to the cultural discourse after watching a certain show. Some interviewees associate terms such as "withdrawals", "overdose," "habit," "functional binger," or "vice" with binge-watching, which illustrates the transposition of concepts from medical and psychological terminology related to addiction in the public discourse about binge-watching. On the other hand, some respondents were proud of their achievement at the end of a marathon viewing session, especially when it comes to the consumption of smart media products, associated with consumer intelligence and superior quality. At the same time, the study found that Netflix's post-play function (which makes the next episode play automatically at the end of the previous one) has an important role in the audience's decision to watch episode after episode (Steiner $\& \mathrm{Xu}, 2018)$.

Shannon-Missal (2013) showed that $81 \%$ of the US users choose to engage in media marathons because it brings them pleasure, $53 \%$ because they want to know what will be going on in the fictional universe, and 37\% because they do not want to be left behind and risk hearing or reading spoilers which could destroy their future viewing experience. The duration of the viewing sessions is another important factor that can help in defining the concept of binge-watching. According to the same study, $25 \%$ of the respondents watched an entire 13-hour season 
in just two days. Some users see a negative connotation in such an activity, while others view it as a positive experience that can help them to temporarily escape the daily course of life.

Pittman and Sheehan (2015) developed the methodology used by Papacharissi and Mendelson (2007) and identified five main motivations for engaging in bingewatching sessions: engagement, relaxation, time occupation, hedonism, and the social component of consumption. According to the research, the most important factor for users choosing to binge-watch is that this type of consumption creates a higher degree of involvement than watching an individual episode. This involvement may result from the superior quality of the productions or from the fact that individuals are more committed to watching a show during marathon sessions and tend to pay more attention to the fictional world. At the same time, the degree of consumer involvement is directly proportional to the frequency of engaging in binge-watching. The more a user feels emotionally involved in the story, the more feelings he develops for the characters, the more he chooses to engage in this type of behavior.

The social aspect also plays an important role. Pittman and Sheehan (2015) have identified many instances where users plan their collective marathon views in advance, suggesting that watching certain programs might become more similar to events like the Super Bowl for some users, somewhat similar to a ritual. Users can also refer to binge-watching as a fortifying experience, during which they suspend their thoughts and obligations from everyday life. In order for the level of consumer engagement to be as high as possible, the narrative universe must be rich enough to allow a complete immersion in a fictional world that psychologically removes them from their daily life. In the same sense, Feeney (2014) notes that some users binge-watch shows as a kind of reward they deserve after a hard day at work. Another interesting observation from Pittman and Sheehan's (2015) study is that $84 \%$ of the 272 respondents - who were selected using a snowball sampling technique - used Netflix to binge-watch shows, and $63 \%$ of them said they preferred this portal because it allowed them to engage in this type of consumption. The second technology in the top preferences was the DVR, with a presence of only $9 \%$. The observation reflects major market trends and the strong connection between Netflix and binge-watching, reinforced by the company's marketing strategies.

Conlin, Billings, and Auverset (2016) examined the relation between a phenomenon called fear-of missing-out (FoMO) - the fear of not taking part in a pleasant activity that other people share -, and the behavior of users who engage in media marathons. According to the authors, the role this psychological phenomenon plays in choosing the pace of watching television shows is very important: "FoMO seems to be driving many of the changes that are evident in modern me- 
dia consumption; part of the enjoyment of watching TV is being able to talk about it with friends, and being part of an overarching, cultural conversation. This is particularly evident in serial dramas, where plot points and twists are likely to be prematurely revealed the longer a person waits to watch current episodes. This behavior was not evident for reality TV shows or sitcoms, where individuals can easily move both in and out of the narrative [...]" (Conlin et al., 2016, 162).

According to the same study, the relation between FoMO and serialized television narratives is tripartite: on the one hand, there is a category of fans who watch weekly episodes as soon as they are broadcast to ensure that no one watches the content or discusses the plot before them. A second category practices binge-watching to catch up with the episodes they missed during the initial broadcast, so they can join the conversation. The third type of user engages in media marathons and consumes content in an accelerated way, possibly out of the fear of missing an essential television show or because they feel pressured by the recommendations of their peers (Conlin et al., 2016, 154-155). In this sense, the authors note, "FoMO could explain how people may opt to binge-watch not because they prefer to, but rather for a presumed psychological need to do so, as not binging results in being excluded from conversational aspects and references of modern society."

In addition to FoMO and the collective dimension of binging TV shows, other key factors that lead to users engaging in this type of behavior are boredom and users' desire to fill their leisure time with something (Merikivi et al., 2016). The cited study also shows that marathon viewing is not necessarily a constant behavior for all users, but is part of a more complex network of ways users interact with the media system. However, users are of the opinion that the opportunity to engage in binge-watching sessions is an important advantage for SVoD services. In this way, all-at-once distribution has direct effects on customers' satisfaction rates and leads to a better retention of the number of subscribers, against their ability to give up the service very easily.

Walton-Pattison, Dombrowski and Presseau (2016) show that users engage in binge-watching sessions at least once a week. Looking at the phenomenon from a psychological perspective, the authors say that automatism, anticipated regret and conflict between goals are important factors that characterize this type of behavior. While automatism may be associated with an extended sedentary behavior, potentially encouraged by the post-play function of SVoD services, anticipated regret refers to those situations where users are conscious that they will regret if they choose to watch several episodes of a series consecutively. The phenomenon is closely related to the conflict between users' goals, which defines the cases in which consumers spend their time binging TV shows instead of pursuing other goals that they could rationally consider more important. 
Matrix (2014) indicates two emerging behavioral patterns of teenagers who binge-watch TV shows. On the one hand, because of the new distribution systems, discussions (around the water cooler) about their favourite shows disappear and are replaced by virtual interactions. The phenomenon through which socializing technologies become part of the consumption mechanism, and the use of SNSs is triggered by TV shows, is called Social TV (Buschow, Schneider, \& Ueberheide, 2014; Gray, Sandvoss, \& Harrington, 2017; Matrix, 2014; Roebuck, 2011). Therefore, the disappearance of next day real-life discussions about what a certain character did or about the ending of a specific episode do not necessarily mean a loss in the cultural discourse, but rather a reconfiguration of these social interactions.

A second observation by Matrix (2014) is the strong link between binge-watching and the content itself. Users prefer to binge on media products that offer them the possibility to immerse themseves in a fictional world and to create strong emotional ties to the characters in the series. The most appreciated shows are the narratively complex ones, which also gain popularity because of their multidimensional, complex characters (Matrix, 2014, 130), in line with the observations of other authors (Lotz, 2017; Tryon, 2015). The audience also chooses to binge-watch media productions to satisfy their intellectual curiosity, for the sexual content, or for a certain production's quality. The social component of watching television shows projects itself into a broad phenomenon, placed at the confluence between the digital distribution of series and the use of social networking sites.

Even though researchers studied the behaviors and motivations of consumers related to binge-watching, as well as the frequency or duration of binge-watching sessions, few studies (Buschow, Schneider, and Ueberheide 2014; Pittman and Tefertiller, 2015) addressed the connection between watching series and social networking sites (SNS) as a means of expression and self-representation used by consumers. "Social TV" (Buschow, Schneider, and Ueberheide, 2014) - the use of social networking sites such as Facebook or Twitter triggered by watching television programs - may be a useful indicator on how consumers interact with these television products but also on the way they relate to their own experiences when they are not in a position to answer the questions of a scientific survey.

From this perspective, the study of user-generated content on SNSs has proven to be a useful tool in examining how users relate to different aspects of reality, such as politics or media representations of certain events (Asur and Huberman, 2010; Bonilla and Rosa, 2015; Hille and Bakker, 2014; van der Meer and Verhoeven, 2013). In this context, analyzing user-generated content on SNSs can be a relevant means of determining how the audience relates intellectually and emotionally to binge-watching. 


\section{Methodology}

This chapter aims to both analyze the emotions and behaviors expressed by users in relation to binge-watching, as well as to examine the correlations between different behavioral patterns and the emotions generated by them.

The main research method used in this paper is content analysis. This is duplicated by individual examples in the dataset in order to understand the connection between binge-watching and certain variables taken into account by the study. Most parts of the analysis were performed automatically, using text analysis and data visualization software: KH Coder, Sketch Engine, Tableau Desktop and Microsoft Excel.

The analyzed data set contains 4089 comments that mention binge-watching, posted on the official Facebook pages of 71 original Netflix productions. All Netflix original productions that had an active Facebook page on June 29, 2018, except for children productions and those in a language other than English, were taken into account. The extracted comments were posted between January 1, 2016 and June 29, 2018. The 4089 analyzed comments were filtered for relevancy (the presence of the word "binge-watching" in its various forms) using Microsoft Excel, out of a total of 220,591 extracted messages. Data extraction took place on June 29, 2018.

The comments were extracted through API queries, using Facepager. All the comments analyzed contain the word "binge" in one of its recurring forms in the corpus: "binge," "binge-" "binged," or "binging." Comments that referred to other behaviors, such as binge-drinking or binge-eating, were manually removed from the data set.

The study used inductive coding (Stuckey, 2015; Thomas, 2006) to categorize behaviors and emotions recurrently expressed by users. After the codes were established, the data went through an automated analysis using KH Coder, a free software for quantitative content analysis and natural language processing. The research also used Sketch Engine, a language corpus management software. With the help of this software, recursive words and expressions were identified in the data set, thus helping to produce the coding file used in the KH Coder automatic quantitative analysis.

The data set also underwent a sentiment analysis, in order to determine the overall attitude of users towards their binge-watching experiences. The sentiment analysis was wade using Text Analysis, a Google Sheets extension for natural language processing and automatic learning. The charts in this paper were created using Tableau Desktop and KH Coder.

In order to identify the recurrent behaviors present in the data set, a coding grid was created by manually analyzing comments and identifying recurring message types. The grid consists of two main categories (Behaviors and Emotions), respectively in two series of subcategories. These will be detailed in Tables 1 and 2 . 
Table 1. Recurrent behaviors identified in the data set

\begin{tabular}{|c|c|}
\hline Behavior & Meaning \\
\hline Past & Comment referring to a past binge-watching session \\
\hline Currently & Comment referring to a present binge-watching \\
\hline Future & Comment referring to a planned binge-watching session \\
\hline Binge re-watch & Comment referring to binge-watching a media product that the user has already viewed \\
\hline Want to binge & Comment expressing the desire to be able to binge-watch a show \\
\hline Habit & $\begin{array}{l}\text { The user defines himself/herself as a binge-watcher or reffers to his/her constant engaging } \\
\text { in binge-watching sessions. }\end{array}$ \\
\hline No binge & $\begin{array}{l}\text { The user tries to avoid binge-watching the show (usually) in order to have something to } \\
\text { watch over a longer period, to avoid spoiling the experience or as a critique towards binge- } \\
\text { watchers. }\end{array}$ \\
\hline Catch-up & The user expressed the desire or the 'need' to catch up with a show. \\
\hline
\end{tabular}

Table 2. Recurrent emotions identified in the data set

\begin{tabular}{|c|c|}
\hline Emotion & Meaning \\
\hline Enjoyment & $\begin{array}{l}\text { The user displays positive affects towards the show and/or his/her behavior. Usually } \\
\text { related to statements about the show, the casting, the acting, etc. }\end{array}$ \\
\hline Engagement & The user clearly states a high level of engagement as a motivation for binge-watching . \\
\hline Bragging & $\begin{array}{l}\text { The user mentions watching several episodes, an entire season or several seasons of the } \\
\text { show during a very short period of time. Usually associated with past or current behaviors. }\end{array}$ \\
\hline Frustration & $\begin{array}{l}\text { The user expresses frustration towards the show, his impossibility to binge-watch or the } \\
\text { fact that he has to wait for a long time between different segments of the show. }\end{array}$ \\
\hline Impatience & The user clearly states his impatience for the next segment of the production to be released. \\
\hline
\end{tabular}

The automatic content analysis using KH Coder was based on recurring syntaxes identified in the data set. Also, the coding file was developed partly manually, partly with the help of Sketch Engine, which was useful in identifying word associations and recurring word structures in the data set. Table 3 shows two code examples used in the KH Coder automated analysis.

Table 3. Examples of codes used in the automated data analysis

\begin{tabular}{ll}
$\begin{array}{l}\text { Behavior, emotion } \\
\text { or item of interest }\end{array}$ & \multicolumn{1}{c}{ Code } \\
\hline $\begin{array}{l}\text { E.g. } 1 \\
\text { Enjoyment }\end{array}$ & $\begin{array}{l}\text { great show | awesome show | amazing show | good show | favorite+show | love | absolutely } \\
\text { loviting | great job | great season | incredible show | excellent | incredible | awesome | best } \\
\mid \text { near(thank-netflix) }\end{array}$ \\
E.g. 2 & dvr | dvring | record | recorded | tape | taping | recording \\
DVR &
\end{tabular}

The automatic coding has also taken into account other elements of interest for this paper, such as mentioning certain technologies closely related to binge-watch- 
ing, the moment of viewing, the length of the segment of the series being viewed, and a series of terms referring to addiction (Steiner and Xu 2018). A similar methodology was used by Radu Meza (2014) to explore discursive patterns in satirical fake news published by Timesnewroman.ro.

A similar methodology was used in one of the author's previously published papers (Boca, 2017), which examined - using an older dataset - behaviors and emotions related to binge-watching expressed by linear television consumers as compared to the fans of digitally-distributed series.

\section{Results and discussion}

The dataset (the entire set of data can be seen in the appendix) shows a tendency of users to refer more often to their own binge-watching sessions when it comes to comedy series (Figure 1). Thus, only 4 of the top 10 productions as a number of generated comments can fit into the tradition of the prime-time series: Orange is the New Black, Stranger Things, The Last Kingdom, and The Crown. Most of the binge-watching sessions are related to comedy series such as Grace and Frankie (417), The Ranch (221) and Trailer Park Boys (179). The phenomenon is all the more interesting when compared to the results of our previous study (Boca, 2017): between 2013 and 2016, 8 out of the top 10 productions which generated comments about binge-watching were one-hour dramas. The observation may suggest that although marathon viewing has become a cultural phenomenon in association with original Netflix dramas, this type of behavior is no longer the exclusive feature of productions in this category. Also, as long as the consecutive consumption of two episodes in a production can be defined as a binging session - according to Netflix official statistics (Netflix Media Center, 2013) - users can become part of the cultural trend of binge-watching (Jenner, 2015) even by watching a series for just one hour (for example, two episodes of Grace and Frankie or Trailer Park Boys).

Comedy fans can therefore express their membership to the binge-watcher group on social networking sites after a shorter session than the one needed to watch multiple episodes of a one-hour drama. The fact that users can accumulate cultural capital by being part of the binge-watching phenomenon, no matter if they watch dramas or comedies, is a strategic advantage for Netflix. As long as consumers have a sense of belonging to a particular privileged group, it will be much more difficult for them to give up on the services provided by the company (Jenner, 2015). And if participation in the cultural phenomenon in question requires little effort, as in the case of comedies, the company's strategic position improves considerably. 


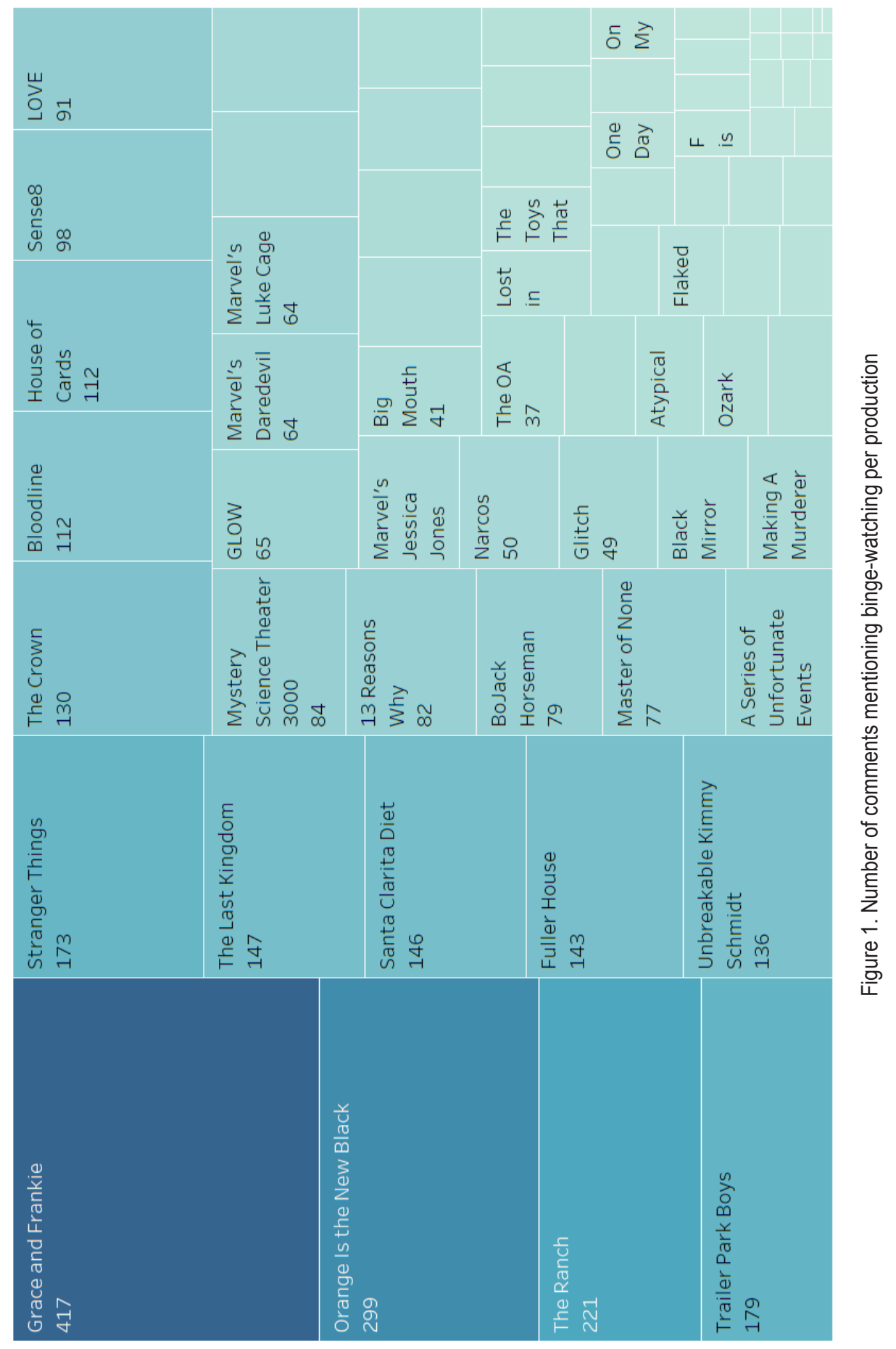




\section{Behavioral patterns}

Examining the dataset in terms of binge-watching related user actions has led to the identification of some interesting behavioral patterns (Figure 2). Most comments refer to binge-watching sessions that took place in the past (25.07\%). Two of the most common types of comments referring to such an activity that took place in the past are those in which users write about the enjoyment they felt while binging on a series (e.g. "Amazing season! Binged it this weekend") and those in which users brag about binge-watching a large segment of the production in a short time, in order to accumulate cultural capital (e.g. "I binge-watched all 5 seasons within the course of a week (what can I say? bad weather and vacation) and am completely hooked.").

Another category of frequently encountered comments refers to users' plans to engage in a marathon viewing session in the future (20.97\%). In many cases, comments like this show a link between binge-watching and weekend, as observed by our previous study (Boca, 2017) (e.g. "I know what I'm binge watching this weekend!").

Examining the data set has also brought to light other types of recurring behavior. $3.83 \%$ of users choose to rewatch series (e.g. "Just finished rewatching season 1. Tomorrow binge re watch season 2. Season 3 here i come"), usually because of the long temporal gap between two seasons of a production or as a way of remembering the characters and actions of the previous season right before Netflix releases a new one (e.g. "Finally! I plan to rewatch S1 before I binge S2. Solid and engaging story and fantastic acting. I'm so excited, I can't wait!").

Another interesting phenomenon is that $2.51 \%$ of the commenters explicitly express their decision not to binge-watch a certain series, but to spread it along a longer period of time. In many cases, they are afraid that a marathon session might alter their experience or that if they view the show in a single sitting, there will be nothing left for them to watch afterwards (e.g. "I am impatiently waiting for the new season. I need OITNB in my life now! Okay so I watch all the old episodes but still! I promise this time I won't binge watch all new episodes in the same night...."). When compared to the results of our previous study (Boca, 2017), which showed that $5.26 \%$ of the users consciously chose not to engage in bingewatching sessions in order not to spoil their experience, the percentage of such comments in the present data set is somewhat lower. This result might suggest that when it comes to Netflix series, binge-watching has evolved from the position of an innovation in TV consumption, sometimes looked upon with suspicion, to the status of a consumption norm accepted by most users.

At the same time, $6.76 \%$ of the users express their desire to be able to engage in a binge-watching-session, especially when they finish watching a season, and waiting for an entire year until the next one will be out does not sound like a pleasant outlook. 


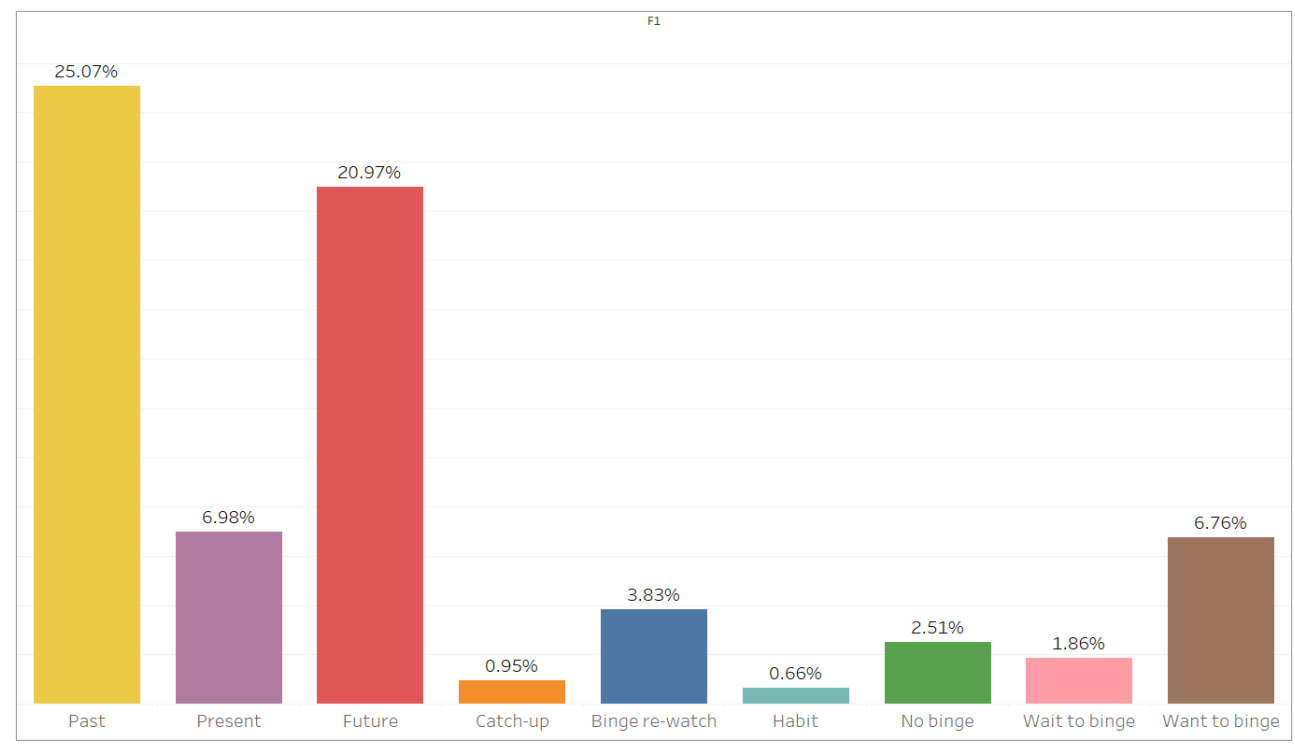

Figure 2. Behavior frequency in the dataset

Expressing behaviors such as waiting until the entire season of a show or even several seasons of a show are out in order to be able to see them in extended watching sessions is usually related to Neflix co-productions (e.g. The Last Kingdom), where the streaming company does not always distribute the episodes all at once, or to the seasons of a series which were released before Netflix bought the production.

\section{Emotional patterns}

Exploring consumer emotions in relation to their binge-watching experiences has led to the identification of five recurring types of manifestation (Figure 3). From this perspective, the most commonly expressed emotion is the pleasure generated by a past or present binge-watching experience (31.88\%). The results confirm the observations made by Pittman and Sheehan (2015), who explored the important role of hedonism and relaxation in users' decision to engage in media marathons.

Another important reason why people binge-watch series is the engagement they feel in relation to the fictional world. The phenomenon appears explicitly in $5.49 \%$ of the comments. The importance of immersion in the narrative universe as regards the audience's decision to watch multiple episodes of the same show in one sitting was highlighted by previous studies (Steiner and Xu, 2018; Pittman and Sheehan, 2015).

Another interesting phenomenon emerging from the data is the large number of comments (13.23\%) in which users brag about their habit of engaging in extend- 
ed and frequent marathon viewing sessions, or about watching a major segment of a series within a short period of time (e.g. "The day it premiered I binged it all in one sitting, best decision I've ever made!"). The mechanism behind comments in this category is related to users' desire to accumulate cultural capital (Jenner, 2015). The link between users' "bragging" and media marathons was also observed by Snyder (2016) and Pittman and Sheehan (2015) and is a clear indication of the fact that binge-watching is a type of consumption that bears positive social connotations for many users (Stoldt, 2013). Thus, watching an ample segment of a series in a short period of time gives consumers the right to boast with their performance and helps them gain a higher status in the community. Such comments clearly mark the strong effect of social pressure on users' decision to engage in extended watching sessions. Also, the fact that fans choose to share all these experiences with the other members of the community confirms the importance of the social component of consumption in the binge-watching phenomenon (Pittman and Sheehan, 2015). The notable presence of such an affective manifestation among digital series consumers is explained by the very essence of the medium, which gives users the possibility to decide their own viewing schedule and watch how many episodes they want when deciding to do so.

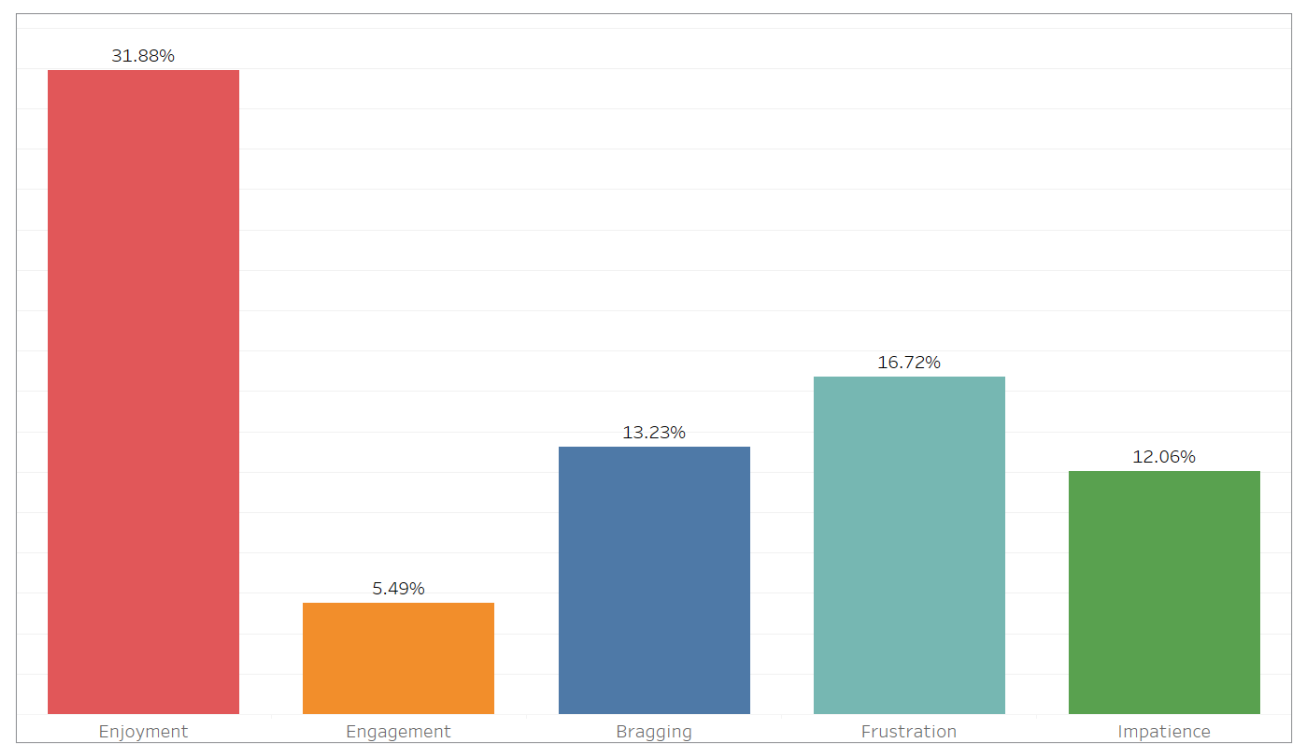

Figure 3. Emotion frequency in the dataset

At the same time, many comments reflect the frustration of users in relation to different aspects of their experiences $(16.72 \%)$. Users usually complain about the long timespan between the releases of two consecutive seasons of a production (e.g. "We so binge watched that to fast and loved every minute of it. I am so 
depressed now that we have to wait !!! Hurry up and make the next season so we can see baby Bennett"). In the same vein, $12.06 \%$ of the users express their impatience towards next season's release date. These observations reveal that although consumers enjoy the benefits of Netflix's distribution mechanisms, such as giving them the opportunity to configure their own viewing program, they are caught on the horns of a dilemma: either they choose to watch episodes in shorter viewing sessions, over a longer period of time, in which case they can not enjoy the benefits of binge-watching, or choose to binge the series, but wait for a whole year until the next season gets released. In this last sense, Jonathan Cohen (2004) examined the nature of the parasocial relations that members of the audience develop with the characters in television series. His study has shown that the emotional reactions that consumers experience when they are deprived of access to their favorite characters - either due to the departure of actors from the production team, due to the death of the character in the fictional universe, or because of the show's cancelling - are as intense as those triggered by a separation that takes place in real life. Given this psychological phenomenon, the frustration felt by consumers when they see themselves facing a long wait until reunion with their favorite characters becomes easier to understand.

\section{Sentiment analysis}

Beyond the concrete affective manifestations identified in the comments analyzed, the dataset was subjected to a sentiment analysis, in order to obtain a clearer picture of the general attitude of the users towards binge-watching. The analysis was performed automatically using Text Analysis, a Google Sheets extension used for natural language processing and automatic learning, based on the AYLIEN Text Analysis API. Such methodological approaches have proven to be useful in the processing large data sets, and help to identify people's opinions, attitudes and feelings in relation to a particular topic (Pak and Paroubek, 2010; Liu, 2012). The analysis method is relevant in the exploration of user-generated content on review sites, forums, micro-blogs, or social networking sites (Liu, 2012).

Results show a predominantly positive attitude towards binge-watching (62\%). The observation shows that this consumer behavior is identified by most users with a constructive experience that has lost the negative connotations held by expressions such as binge-drinking or binge-eating. The phenomenon was previously discussed by Ryan Stoldt (2013).

\section{Behavior and emotion co-occurrences}

In order to observe how behaviors and emotions are connected, as well as to identify the contexts in which other elements of interest identified in dataset tend to appear, the comments went through an automatic coding process using $\mathrm{KH}$ 
Coder. In addition to the behaviors and affects already explored in the present study, the coding agenda has also considered other relevant aspects: the specified series segment (season or episode) as an indicator of the duration that users associate with binge-watching, the weekends as the prevailing days when the audience chooses to engage in binge-watching sessions and a series of terms related to addiction. The calculation of the co-occurrence matrix was done automatically and is based on the Jaccard similarity index.

The co-occurrence network shown in Figure 4 provides a view of the similarities between different codes. Thicker lines denote a close connection between two elements, while clusters of a certain color indicate a high level of co-occurrence of the target codes.

Frustration - Netflix - addiction. Cluster 1 indicates a high level of co-occurence between comments expressing users' frustration, terms related to addiction and mentioning Netflix. As shown before, in many cases subscribers get frustrated because of the long wait between two consecutive seasons of a production. As a result, people try to approach Netflix representatives through the comment sections on series' Facebook pages, asking them to release the next season of a particular series as soon as possible. In a similar context, many users tend to express their frustration at the end of a season by using terms related to addiction, such as " $\mathrm{I}$ ' $\mathrm{m}$ with withdrawals" or "I'm addicted to this show."

Binge-watching - Season - Past - Bragging - Enjoyment. The high degree of cooccurrence between the concept of binge-watching and the mention of a specific season of a production indicates that many users associate this behavior with the consumption of an entire season. The phenomenon is even more noticeable in the case of consumers who brag about their performance of watching a large segment of a show over a short time. In most cases, their claims are made in connection with a freshly released season. Also, many of those who express their enjoyment in relation to their binge-watching experiences that have taken place in the past are mostly doing so as a result of finishing a season of a production. All these comments are closely related to the feeling of fulfillment that users have after watching a season, a phenomenon identified by Steiner and $\mathrm{Xu}$ (2018).

Future - Weekend. Cluster 3 indicates a strong correlation between future plans to engage in binge-watching sessions and weekends. Many users engage in this consumer behavior either as a reward after a difficult week at work (Feeney, 2014), or as an opportunity to spend a relaxing and pleasant time in the company of close people (Pittman and Sheehan 2015). At the same time, many of them refer to the marathon viewing sessions that took place over the previous weekend, often expressing pride in connection with watching a whole series or a whole season over a short timespan. 


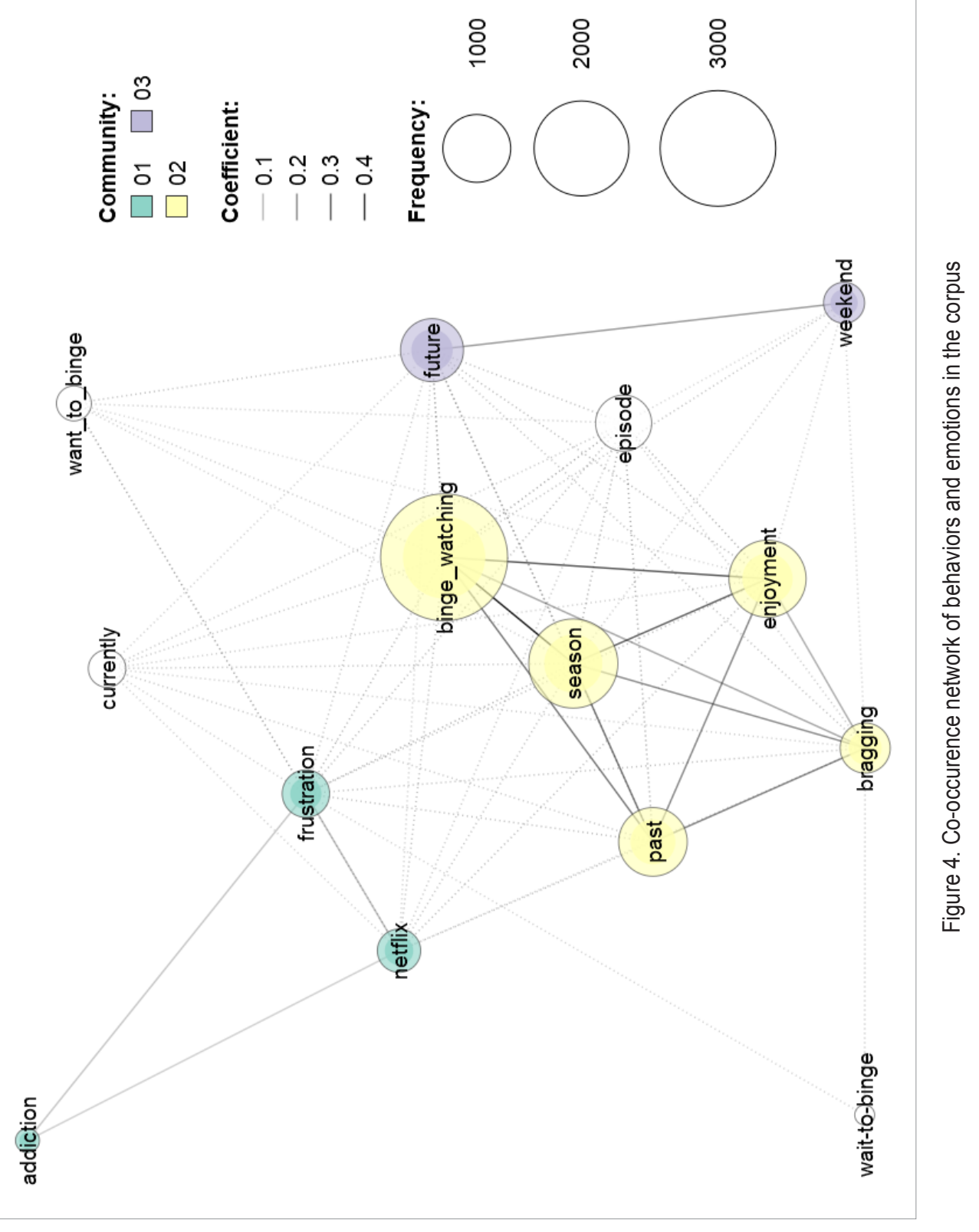




\section{Conclusion}

The study identified a number of specific behaviors and emotions that appear recurrently when users refer to their own binge-watching sessions. Some talk about the pleasure that their recent media marathons have brought them, and others plan ahead for the perfect binge-watching day or weekend. Also, many users choose to rewatch certain media products either to relive the immersion in the fictional universe (Steiner and Xu, 2018; Pittman and Sheehan, 2015), or to recall the important elements of the story before a new season gets released.

Another relevant behavior is that of users who engage in marathon viewing sessions to catch up with the segments of productions they missed, a manifestation closely related to the FoMO phenomenon (Conlin et al., 2016), and the social pressure which in many cases determines users' participation in extended viewing sessions.

Moreover, an interesting emotional pattern is revealed by the comments in which users express their frustration with the distribution mode: this frustration is usually generated by the long waiting times between two consecutive seasons of the same production.

The extracted data also showed that many consumers brag about the consumption of major segments of the series in short intervals, a phenomenon previously noticed by Pittman and Sheehan (2015), but also by Snyder (2016). Such behavior gives users the opportunity to accumulate cultural capital and to gain a privileged position in the community.

The sentiment analysis of the corpus has shown that most users refer to marathon viewing as a positive experience that no longer has the negative medical and psychological connotations of phrases such as "binge-drinking" or "binge-eating" (Stoldt, 2013). On the contrary, binge-watching seems to be invested with a major social capital, while consumers talk proudly about their extended watching sessions and use them to self-represent in interactions with other members of the digital communities.

In the same broad perspective, it is interesting that many users associate comedy series with binge-watching, despite the public and scientific discourse that this type of media behavior is inextricably linked to prime-time dramas. A first psychological mechanism that may be behind this divergence is that, in the face of a sociological questionnaire, consumers would be prone to say they usually watch media products associated with a high degree of intelligence and education. The key to a second possible explanation could be that binge-watching a drama involves both a higher concentration effort and more time. However, as long as the consecutive watching of two episodes of a production can be defined as binge-watching (Netflix Media Center, 2013), regardless of their genre or duration, comedy fans can enter the public discourse and accumulate cultural capital in the 
same way that the fans of prime-time drama do it, with the only exception that in their case the effort required in order to gain the right to express their adherence to the privileged group of binge-watchers is considerably smaller.

At the same time, exploring the internal mechanisms of binge-watching through user-generated content on Facebook has shown that this type of approach can be a key element in understanding certain media consumption habits. In addition to sociological research based on questionnaires, analyzing data extracted from social networking sites can be a useful tool in decoding current media consumption patterns. At the same time, the advantage of such a methodological approach is that it removes the need for a sociological tool such as the questionnaire, which could lead to the self-censorship of the questioned consumers.

Thus, this study identifies a number of negative emotions associated with binge-watching as well as a strong tendency of $\mathrm{VoD}$ users to boast with their watching performance to their peers. At the same time, studying how consumers self-represent on social networking sites revealed a strong correlation between comedy series and binge-watching, a result which partially contradicts other papers indicating a close connection between this type of behavior and prime-time dramas.

\section{References}

1. ARRIS Consumer Entertainment Index. (2015).

2. Asur, S., \& Huberman, B. A. (2010). Predicting the Future with Social Media. 2010 IEEE/WIC/ACM International Conference on Web Intelligence and Intelligent Agent Technology, 1, 492-499.

3. Boca, P. (2017). Binge-Watchers. Behavior Patterns and Emotions. Studia Universitatis Babeș-Bolyai Ephemerides, (2), 5-27.

4. Bonilla, Y., \& Rosa, J. (2015). \#Ferguson: Digital protest, hashtag ethnography, and the racial politics of social media in the United States. American Ethnologist, 42(1), 4-17.

5. Buschow, C., Schneider, B., \& Ueberheide, S. (2014). Tweeting television: Exploring communication activities on Twitter while watching TV. Communications, 39(2), 129-149.

6. Cohen, J. (2004). Parasocial Break-Up from Favorite Television Characters: The Role of Attachment Styles and Relationship Intensity. Journal of Social and Personal Relationship, 21(2), 187-202.

7. Conlin, L., Billings, C., \& Auverset, L. (2016). Time-shifting vs. appointment viewing: the role of fear of missing out within TV consumption behaviors. Communication \& Society, 29(4), 151-162.

8. Deloitte. (2019). 2019 Media \& Entertainment Industry Outlook.

9. Feeney, N. (2014). When, Exactly, Does Watching a Lot of Netflix Become a "Binge"? The Atlantic. 
10. Gray, J., Sandvoss, C., \& Harrington, C. L. (2017). Fandom: identities and communities in a mediated world.

11. Hille, S., \& Bakker, P. (2014). Engaging the Social News User. Journalism Practice, $8(5), 563-572$.

12. Hiller, R. S. (2017). Profitably Bundling Information Goods: Evidence From the Evolving Video Library of Netflix. Journal of Media Economics, 30(2), 65-81.

13. Jenner, M. (2015). Binge-watching: Video-on-demand, quality TV and mainstreaming fandom. International Journal of Cultural Studies, 20(3), 304-320.

14. Karve, A. (2018). Bollywood Streaming Service Looks Past Netflix Model for Growth - Bloomberg. Retrieved July 27, 2018, from Bloomberg website: https:// www.bloomberg.com/news/articles/2018-06-04/bollywood-streaming-servicelooks-past-netflix-model-for-growth

15. Katz, E., Blumler, J. G., \& Gurevitch, M. (1974). Uses and Gratifications Research. Public Opinion Quarterly, 37(4), 509.

16. Levy, M. R., \& Windahl, S. (1984). Audience Activity and Gratification. A Conceptual Clarification and Exploration. Communication Research, 11(1), 51-78.

17. Liu, B. (2012). Sentiment Sentiment Analysis Analysis and and Opinion Opinion Mining Mining. Synthesis Lectures on Human Language Technologies, 5(1), 1-167.

18. Lotz, A. D. (2014). The Television Will Be Revolutionized, Second Edition (2 edition). New York: NYU Press.

19. Lotz, A. D. (2017). Portals: A Treatise on Internet-Distributed Television. Maize Books.

20. MacroTrends. (2019). Netflix Revenue 2006-2019. Retrieved May 28, 2019, from MacroTrends.net website: https://www.macrotrends.net/stocks/charts/NFLX/netflix/revenue

21. Matrix, S. (2014). The Netflix Effect: Teens, Binge Watching, and On-Demand Digital Media Trends. Jeunesse: Young People, Texts, Cultures, 6(1), 119-138.

22. McNamara, M. (2012). The side effects of binge television. Retrieved August 1, 2018, from LA Times website: http://articles.latimes.com/2012/jan/15/entertainment/la-ca-netflix-essay-20120115

23. Merikivi, J., Mäntymäki, M., Salovaara, A., \& Zhang, L. (2016). BINGE WATCHING TELEVISION SHOWS: CONCEPTUALIZATION AND MEASUREMENT. Research Papers, (16).

24. Meza, R. (2014). Discursive Patterns in Fake Online News. An Analysis of Timesnewroman.Ro Articles Over Five Years. Studia Universitatis Babeș-Bolyai Ephemerides, 59(2), 59-80.

25. Mittell, J. (2015). Why has TV storytelling become so complex? Retrieved August 5, 2018, from The Conversation website: https://theconversation.com/why-has-tvstorytelling-become-so-complex-37442

26. Netflix Media Center. (2013). Netflix Declares Binge Watching is the New Normal. Retrieved July 30, 2018, from https://media.netflix.com/en/press-releases/netflixdeclares-binge-watching-is-the-new-normal-migration-1 
27. Newman, M. Z. (2014). Video revolutions: on the history of a medium. Columbia University Press.

28. Oxford Dictionaries. (2013). Word of the Year 2013 - Shortlist. Retrieved September 13, 2018, from Oxford Dictionaries website: https://en.oxforddictionaries.com/ word-of-the-year/shortlist-2013

29. Oxford English Dictionaries. (n.d.). binge-watch | Definition of binge-watch in English by Oxford Dictionaries. Retrieved July 30, 2018, from https:// en.oxforddictionaries.com/definition/binge-watch

30. Pak, A., \& Paroubek, P. (2010). Twitter as a Corpus for Sentiment Analysis and Opinion Mining. Proceedings of the Seventh Conference on International Language Resources and Evaluation (LREC'10), 1320-1326.

31. Papacharissi, Z., \& Mendelson, A. (2007). An Exploratory of Reality Appeal: Uses and Gratifications of Reality TV Shows. Journal of Broadcasting E Electronic Media, 51(2), 355-370.

32. Pittman, M., \& Sheehan, K. (2015). Sprinting a media marathon: Uses and gratifications of binge-watching television through Netflix. First Monday, 20(10).

33. Pittman, M., \& Tefertiller, A. C. (2015). With or without you: Connected viewing and co-viewing Twitter activity for traditional appointment and asynchronous broadcast television models. First Monday, 20(7).

34. Roebuck, K. (2011). Social TV: high-impact strategies - what you need to know: definitions, adoptions, impact, benefits, maturity, vendors. Lighting Source.

35. Rubin, A. M. (1983). Television uses and gratifications: The interactions of viewing patterns and motivations. Journal of Broadcasting, 27(1), 37-51.

36. Shannon-Missal, L. (2013, December 18). Americans Taking Advantage of Ability to Watch TV on Their Own Schedules.

37. Snyder, R. (2016). Binge On: The Phenomenon of Binge Watching. La Salle University Digital Commons.

38. Statista. (2018). Top internet companies: global market value 2018. Retrieved May 28, 2019, from https://www.statista.com/statistics/277483/market-value-of-the-largest-internet-companies-worldwide/

39. Steiner, E., \& Xu, K. (2018). Binge-watching motivates change. Convergence: The International Journal of Research into New Media Technologies, 135485651775036.

40. Stoldt, R. G. (2013). The behavioral effects of the binge-watching mediamorphosis. Wichita State University.

41. Stuckey, H. (2015). The second step in data analysis: Coding qualitative research data. Journal of Social Health and Diabetes, 3(1), 7. doi: 10.4103/2321-0656.140875

42. Sweney, M. (2018). UK-based sport streaming service adopts Netflix model after \$1bn deal | Media | The Guardian. Retrieved July 27, 2018, from The Guardian website: https://www.theguardian.com/media/2018/may/14/streaming-servicedazn-netflix-sport-us-boxing-eddie-hearn

43. The Aspen Institute. (2015). Netflix and the Growing Trend of "Binge-Watching." 
44. Thomas, D. R. (2006). A General Inductive Approach for Analyzing Qualitative Evaluation Data. American Journal of Evaluation, 27(2), 237-246. doi: 10.1177/1098 214005283748

45. Tryon, C. (2015). TV got better: Netflix's original programming strategies and binge viewing. Media Industries Journal, 2(2), 104-116.

46. van der Meer, T. G. L. A., \& Verhoeven, P. (2013). Public framing organizational crisis situations: Social media versus news media. Public Relations Review, 39(3), 229-231.

47. Walton-Pattison, E., Dombrowski, S. U., \& Presseau, J. (2016). 'Just one more episode': Frequency and theoretical correlates of television binge watching. Journal of Health Psychology, 23(1), 17-24.

48. Wayne, T. (2014). Life Is Streaming Past You. Retrieved September 13, 2018, from The New York Times website: https://www.nytimes.com/2014/05/04/fashion/online-binge-media-culture-finds-a-receptive-audience-in-americans.html

49. Welch, C. (2013). Netflix breaking from all-at-once release strategy with first kids series "Turbo Fast" - The Verge. Retrieved July 27, 2018, from The Verge website: https://www.theverge.com/2013/12/3/5170230/netflix-breaking-from-all-at-oncerelease-strategy-with-turbo-fast

50. West, K. (2014). Amazon Studios Director Explains Decision Not To Take Netflix's All-At-Once Approach With Original Series. Retrieved July 27, 2018, from Cinema Blend website: https://www.cinemablend.com/television/Amazon-StudiosDirector-Explains-Decision-Take-Netflix-All-Once-Approach-With-Original-Series-60427.html 


\section{Appendix}

Number of comments mentioning binge-watching identified on each Facebook page.

\begin{tabular}{|c|c|c|}
\hline Production & Year released & Number of comments \\
\hline 13 Reasons Why & 2017 & 82 \\
\hline A Series of Unfortunate Events & 2017 & 67 \\
\hline Abstract: The Art of Design & 2017 & 1 \\
\hline Altered Carbon & 2018 & 40 \\
\hline American Vandal & 2017 & 2 \\
\hline Arrested Development & 2003 & 23 \\
\hline Atypical & 2017 & 30 \\
\hline Big Mouth & 2017 & 41 \\
\hline Black Mirror & 2011 & 45 \\
\hline Bloodline & 2015 & 112 \\
\hline Boardwalk Empire & 2018 & 3 \\
\hline BoJack Horseman & 2014 & 79 \\
\hline Castlevania & 2017 & 5 \\
\hline Chef's Table & 2015 & 13 \\
\hline Daughters of Destiny & 2017 & 1 \\
\hline Dear White People & 2017 & 14 \\
\hline The Defenders & 2017 & 38 \\
\hline Disjointed & 2017 & 58 \\
\hline Everything Sucks! & 2018 & 18 \\
\hline Evil Genius & 2018 & 10 \\
\hline $\mathrm{F}$ is for Family & 2015 & 13 \\
\hline Fire Chasers & 2017 & 3 \\
\hline Flaked & 2016 & 22 \\
\hline Flint Town & 2018 & 10 \\
\hline Friends from College & 2017 & 14 \\
\hline Fuller House & 2016 & 143 \\
\hline Girlboss & 2017 & 19 \\
\hline Glitch & 2015 & 49 \\
\hline GLOW & 2017 & 65 \\
\hline Grace and Frankie & 2015 & 417 \\
\hline Gypsy & 2017 & 6 \\
\hline Haters Back Off & 2016 & 25 \\
\hline House of Cards & 2013 & 112 \\
\hline Lady Dynamite & 2016 & 8 \\
\hline
\end{tabular}




\begin{tabular}{|c|c|c|}
\hline Production & Year released & Number of comments \\
\hline Lost in Space & 2018 & 27 \\
\hline Love & 2016 & 91 \\
\hline Making A Murderer & 2015 & 42 \\
\hline Marco Polo & 2014 & 25 \\
\hline Marvel's Daredevil & 2015 & 64 \\
\hline Marvel's Iron Fist & 2017 & 41 \\
\hline Marvel's Jessica Jones & 2015 & 50 \\
\hline Marvel's Luke Cage & 2016 & 64 \\
\hline Marvel's The Punisher & 2017 & 37 \\
\hline Master of None & 2015 & 77 \\
\hline Mindhunter & 2017 & 57 \\
\hline Mystery Science Theater 3000: The Return & 2017 & 84 \\
\hline Narcos & 2015 & 50 \\
\hline On My Block & 2018 & 16 \\
\hline One Day at a Time & 2017 & 17 \\
\hline Orange Is the New Black & 2013 & 299 \\
\hline Ozark & 2017 & 29 \\
\hline Santa Clarita Diet & 2017 & 146 \\
\hline Sense8 & 2015 & 98 \\
\hline Seven Seconds & 2018 & 29 \\
\hline She's Gotta Have It & 2017 & 24 \\
\hline Shot In The Dark & 2017 & 17 \\
\hline Stranger Things & 2016 & 173 \\
\hline The Confession Tapes & 2017 & 3 \\
\hline The Crown & 2016 & 130 \\
\hline The Keepers & 2017 & 32 \\
\hline The Killing & 2011 & 3 \\
\hline The Last Kingdom & 2015 & 147 \\
\hline The OA & 2016 & 37 \\
\hline The Ranch & 2016 & 221 \\
\hline The Resident & 2017 & 26 \\
\hline Trailer Park Boys & 2001 & 179 \\
\hline Ugly Delicious & 2018 & 4 \\
\hline Unbreakable Kimmy Schmidt & 2015 & 136 \\
\hline White Rabbit Project & 2016 & 18 \\
\hline Wild Wild Country & 2018 & 9 \\
\hline Wormwood & 2017 & 7 \\
\hline
\end{tabular}

